\title{
Evaluación de las metodologías de enseñanza-aprendizaje de la bioética en las facultades de odontología brasileñas
}

\author{
C.A.S. Garbin, P.E. Gonçalves, A.J.I. Garbin, S.A.S. Moimaz
}

Introducción. La bioética está presente en la odontología y en las otras áreas de la salud, tanto en las investigaciones científicas, que favorecen el progreso de la profesión, como en el propio tratamiento odontológico. Materiales y métodos. El estudio consiste en la evaluación de las metodologías de enseñanza-aprendizaje de la bioética en las facultades de odontología brasileñas. La recolección de datos se realizó mediante cuestionarios semiestructurados enviados por correo electrónico y por correo postal. Se empleó el análisis de contenido por la técnica de análisis de categorías, conforme a lo preconizado por Bardin. Resultados. Entre las 182 facultades de odontología activas en Brasil, apenas 57 (31,3\%) presentaban en su malla curricular la asignatura de bioética. Se observó que la asignatura se enseña generalmente de forma teórica $(77,8 \%)$. Las principales formas de evaluación son: prueba escrita (100\%) y seminarios y/o trabajo en grupo (75\%). Apenas el 6,4\% de los profesores emplean referencias bibliográficas sobre bioética dirigidas específicamente a la odontología. La mayoría de los entrevistados (74,2\%) consideró que la bioética se relaciona de forma directa o indirecta con todas las otras asignaturas. Con respecto a la importancia de la bioética en la formación del cirujano dentista, el 64,7\% apuntó a la relación profesional-paciente. Conclusión. La bioética presenta una metodología de enseñanza y prácticas de evaluación conservadoras, haciéndose necesarias otras metodologías dirigidas para la reflexión de los problemas actuales en el área de odontología, que contribuyen significativamente a la formación integral del cirujano dentista.

Palabras clave. Bioética. Educación superior. Odontología.

\section{Evaluation of teaching-learning methods} of bioethical on Brazilian dental schools

Introduction. Bioethics is present in Dentistry and other health areas, as scientific research that results on profession progress, as in proper clinical attendance. The study consists on valuation of teaching-learning methods of Bioethical on Brazilian Dental Schools. Materials and methods. Data collect occurred by semi-structured questioners send by e-mail and correspondence. It was realized descriptive analysis of quantitative answers, and for qualitative answers, it was used content analysis, by categorical analysis technique in according to Bardin. Results. Among 182 Dental Schools actives in Brazil, only 57 (31.3\%) showed bioethical discipline in its curricular grid. It was observed the discipline is teaching on theoretical form (77.8\%). Principal forms of evaluation are: writing prove (100\%) and seminaries (75\%). Just 6.4\% of professors use bibliographic references about bioethical faced to Odontology specifically. The majority of interviewed person (74.2\%) considered that bioethics is related on the direct and indirect form with all others disciplines. In relation to importance of Bioethical on dental surgeon formation, 64.7\% emphasized it in professional-patient relation. Conclusion. The Bioethics show teaching and practice method of conserver evaluation, and so, it's necessary others methods directed to reflection of actual problems in odontology area that contribute significantly on integral formation of dental surgeon.

Key words. Bioethics. Dentistry. Higher education.
Facultad de Odontología de Araçatuba. UNESP. Araçatuba, São Paulo, Brasil.

Correspondencia

Dra. Cléa Adas Saliba Garbin. Departamento de

Odontología Infantil y Social. Facultad de Odontología de Araçatuba. UNESP. Rua José Bonifácio, 1193. Caixa Postal 341

CEP 16015-050.

Araçatuba, São Paulo, Brasil

E-mail

cgarbin@foa.unesp.br 


\section{Introducción}

En las directrices curriculares brasileñas de 2002, los contenidos esenciales para el curso de Odontología están divididos en contenidos que involucran las Ciencias Biológicas y de la Salud, las Ciencias Humanas y Sociales, y las Ciencias Odontológicas. En las Ciencias Sociales y Humanas se incluyen 'los contenidos referentes a las diversas dimensiones de la relación individuo/sociedad, ya que contribuyen a la comprensión de los determinantes sociales, culturales, comportamentales, psicológicos, ecológicos, éticos y legales, en los niveles individual y colectivo, del proceso salud-dolencia' [1]. En ese campo se insiere la bioética, que es objeto de estudio de este trabajo. La bioética trae el pluralismo inherente a ella, una vez que la visión de lo que es 'bueno' o 'malo' recibe enérgicas influencias de la cultura, de la religión, de las situaciones en las diferentes épocas y hasta de la individualidad. Así, la enseñaza de la bioética hace aflorar los conflictos que los eventos humanos producen, investigándolos para establecer sus jerarquías de valores, incluso de forma autónoma dentro de lo posible [2].

Se sabe que la educación superior asume la formación de competencias, preparando al profesional de la salud para la construcción de su conocimiento, conduciéndolo de manera continua hacia una formación integral [3]. El profesor, en este contexto, presenta un papel fundamental porque además de transmitir informaciones influirá en la formación del futuro profesional por medio de su experiencia y de sus actitudes, y que pasan por la convivencia entre ellos durante las actividades de enseñaza-aprendizaje [4]. Ante esto, las prácticas pedagógicas están sujetas a un análisis crítico y, consecuentemente, pasan por un proceso de renovación y reciclaje, a una mejor fijación de los conocimientos y la formación de recursos humanos. Juzgar, examinar críticamente y hacer recomendaciones es crear condiciones para el desarrollo de la calidad educacional [5-7].

El estudio consiste en una evaluación de las metodologías de enseñanza-aprendizaje de la bioética en las facultades de odontología brasileñas.

\section{Materiales y métodos}

Este estudio es del tipo exploratorio, descriptivo y transversal.
Inicialmente se realizó una investigación en la web del Ministerio de la Educación y Cultura brasileño (MEC), más precisamente en la web del Instituto Nacional de Estudios e Investigaciones Educacionales (INEP), para el conocimiento del número de cursos de Odontología en funcionamiento en Brasil y sus respectivas instituciones de enseñanza superior (IES).

Para confirmar la existencia de la asignatura de Bioética, se entró en todos los sitios institucionales en búsqueda de la matriz o malla curricular. En las instituciones en las que no fue posible por ese medio, se contactó con sus coordinadores para resolver las dudas. Para saber algo al respecto de la existencia de la asignatura en la institución, se realizaron tres tentativas por correo electrónico y dos por correo ordinario, a fin de alcanzar a toda la población en estudio. Al coordinador se le envió una carta exponiendo los objetivos de la investigación y solicitando el permiso para entrar en contacto con los docentes de la asignatura estudiada. El período de obtención de los datos fue desde agosto de 2007 hasta febrero de 2008.

Los datos se recogieron mediante la elaboración y aplicación de un cuestionario; éste contenía preguntas abiertas y cerradas, lo que De Ketele y Roegiers [8] designan como cuestionario de averiguación: 'un estudio de un tema concreto junto a una población, a fin de precisar ciertos parámetros'.

Atendiendo a la Resolución 196/96 del Consejo Nacional de la Salud y sus resoluciones complementarias, el proyecto fue sometido y aprobado por el Comité de Ética en Investigación de la Facultad de Odontología de Araçatuba (UNESP). Junto al instrumento de recogida de datos se remitió el consentimiento informado, el cual aclaraba los objetivos, la metodología, el compromiso de ocultar las identidades (a pesar de no ser solicitada la identificación de los participantes) y la publicación de los datos obtenidos con el presente trabajo.

Los datos obtenidos fueron informatizados. Para tal uso se elaboró una base datos, utilizándose el programa EpiInfo 3.2 para Windows; por medio de éste se obtuvo la frecuencia de los datos referentes a las preguntas cerradas. Para las preguntas abiertas se efectuó el análisis de contenido preconizado por Bardin [9] mediante la técnica de categorización temática. Ésta define las categorías como rúbricas o clases, las cuales reúnen a un grupo bajo un título general. Las respuestas fueron leídas para la identificación de los problemas 


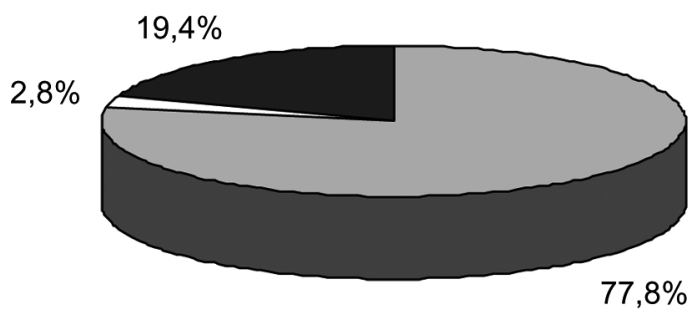

$$
\begin{aligned}
& \text { Teórica } \\
& \square \text { Práctica } \\
& \text { - Teórica y práctica }
\end{aligned}
$$

Figura 1. Distribución porcentual de la metodología empleada en la asignatura de bioética en las facultades de odontología brasileñas, según los docentes entrevistados.

apuntados y el conjunto de ellos formó una malla temática de categorías de análisis para la lectura transversal de todos las declaraciones, o sea, cada relato se leyó y clasificó según las categorías listadas [10].

\section{Resultados}

Se verificó que hay 182 facultades de odontología activas en Brasil, o sea, facultades que presentan el curso de Odontología en su actividad.

En principio se enviaron 180 correos electrónicos, lo que corresponde a todas las facultades que presentaban contacto con el coordinador del curso por internet. A ellos se les remitió una carta sobre el trabajo y el instrumento de recogida de datos, que debería entregarse al profesor responsable de la asignatura estudiada. Se realizaron tres intentos por medio de este recurso. Simultáneamente se enviaron sendas cartas a aquellas instituciones que no tenían ningún tipo de dirección de correo electrónico. Hubo el retorno de 62 correos electrónicos $(34,5 \%)$ y de una carta (50\%). Después de estas tentativas, se realizaron otras dos más por carta para aquellas instituciones que no habían respondido, sumando 109 cartas, y de éstas apenas se obtuvieron 10 cartas de respuesta $(9,2 \%)$. El total de respuestas a la investigación fue del $40,1 \%$.

Entre las facultades analizadas $(n=182)$, apenas $57(31,3 \%)$ presentaban en su malla curricular la asignatura de bioética (23,7\% privadas y 54,3\% públicas). De éstas, en sólo un 54,4\% la nomen- clatura de la asignatura era 'Bioética'. En el 46,4\% restante, la asignatura se denominaba 'Ética Profesional', 'Odontología Forense y Bioética', 'Ética Profesional y/o Odontología Forense, 'Salud Bucal Colectiva', 'Orientación Profesional', 'Odontología Preventiva y Social' y 'Seminarios Integrados'. Del total de 57 instituciones, se logró obtener la respuesta de 38 IES (66,7\%).

Con respecto a los docentes que imparten esta asignatura, un $56,1 \%$ son hombres y un $95 \%$ son graduados en Odontología. El restante 5\% constituyen teólogos o abogados. En la mayoría de los cursos la asignatura es impartida por un único profesor (76,9\%).

En relación a las metodologías empleadas en la asignatura, se observó que ésta generalmente se imparte de forma teórica $(77,8 \%)$ (Fig. 1), utilizando como estrategia de enseñanza la clase teórica $(80,6 \%)$ y seminarios o trabajos en grupo $(69,4 \%)$.

Las principales formas de evaluación del aprendizaje son: prueba escrita (100\%) y seminarios o trabajos en grupo (75\%) (Fig. 2).

Apenas el 6,4\% de los profesores emplea referencias bibliográficas sobre bioética dirigidas específicamente a la odontología, aunque todos los docentes investigados usan bibliografías de autores de renombre, de interés en el área médica y en la salud pública.

La mayoría de los entrevistados $(74,2 \%)$ consideró que la bioética se relaciona de forma directa o indirecta con todas las otras asignaturas de la graduación, tanto básicas como clínicas.

Con relación a la importancia de la bioética en la formación del cirujano dentista, un $64,7 \%$ 


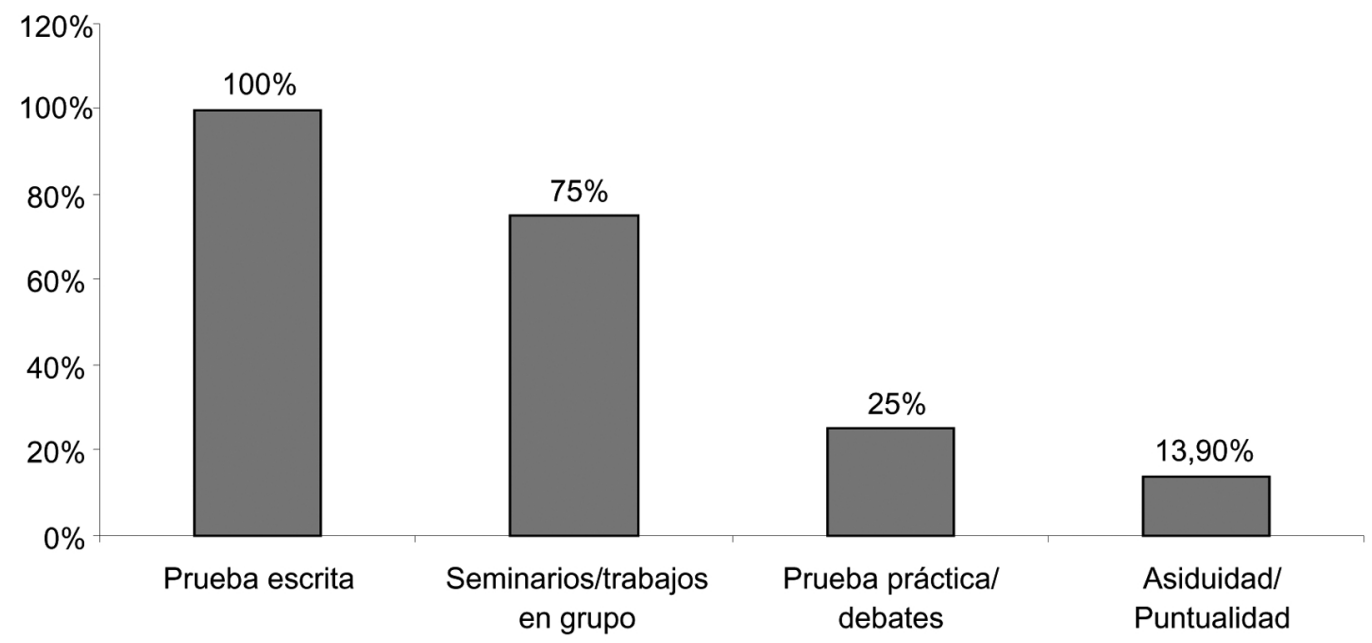

Figura 2. Distribución porcentual de las formas de evaluación empleadas en la asignatura de bioética en las facultades de odontología brasileñas, según los docentes entrevistados. La suma del porcentaje puede ser mayor del 100\% porque la misma respuesta podría clasificarse en más de una categoría. La frecuencia relativa se calculó sobre el total de 36 participantes.

enfatizó la enseñanza de la misma en la relación profesional-paciente (Tabla).

\section{Discusión}

Un proceso de investigación que usa la técnica de averiguación presenta como desventaja 'el pequeño porcentaje de los cuestionarios que se devuelven' [11]. Según lo observado en este estudio, apenas un $40,1 \%$ de las instituciones de enseñaza respondió a la investigación. El envío por correo electrónico fue el más utilizado.

En cuanto a la devolución de los cuestionarios respondidos, Fox [12] relata que pocas veces el número de cuestionarios devueltos sobrepasa los $50 \%$ y, en particular, en la investigación social no es mayor de un 30\%. Ya Pinheiro y Silva [13] obtuvieron una tasa de respuesta del $30 \%$ y Zilbovicius [14], en su estudio con el envío de cuestionarios a las Secretarías de la Salud del Estado de São Paulo, obtuvo una respuesta del 58,4\%. En este estudio, el porcentaje de cuestionarios respondidos fue del $40,1 \%$.

Santos y Rumel [15] enviaron 6.000 cuestionarios por vía postal a los cirujanos dentistas del Estado de Santa Catarina, de los cuales $506(8,43 \%)$ fueron devueltos. Un porcentaje semejante se observó en esta investigación, pues de los 109 cuestionarios enviados vía postal, hubo apenas respuesta en un $9,2 \%$.

Entre las facultades que presentaban la enseñanza de la bioética $(n=57)$, se logró obtener la respuesta de 38 IES (66,7\%).

La enseñanza de la bioética en odontología debe ser esencial en la formación profesional, siendo incompatible la enseñanza de forma aislada, por medio de metodología convencional [16]. Educar es el aprendizaje recíproco entre el profesor y el alumno en el proceso de enseñanza. Sin embargo, se observó en este estudio que el formato de la asignatura es predominantemente teórico, lo que permite pensar que el estudio de la bioética no se está realizando de manera correcta en las Facultades de Odontología [17]. La bioética propiamente no se enseña, se considera como una reflexión, discusión e interacción entre personas dispuestas a discutir y establecer jerarquías de valores relacionados con la salud. La bioética constituye un conjunto de conocimientos que, dirigidos hacia la reflexión y decisión ética, presenta un continuo y rápido desarrollo [18,19].

Así, el estudio profundo de la bioética, tratada como una asignatura de reflexión -diferente de otras enseñanzas dirigidas al desarrollo de capaci- 
Tabla. Distribución de la frecuencia absoluta y relativa según la importancia de la asignatura de bioética en la formación del cirujano dentista, según los docentes entrevistados.

\begin{tabular}{lcc}
\hline Categorías & $n$ & $\% 0^{\text {a }}$ \\
\hline $\begin{array}{l}\text { Relación profesional-paciente (en razón de sus principios, información, } \\
\text { beneficencia, justicia, dirigen las relaciones cirujano dentista/paciente) }\end{array}$ & 22 & 64,7 \\
\hline $\begin{array}{l}\text { Interdisciplinariedad (formar profesionales críticos, reflexivos, humanizados, y que } \\
\text { puedan contribuir a transformar la realidad, principalmente en el contexto de la salud) }\end{array}$ & 13 \\
\hline $\begin{array}{l}\text { Responsabilidad profesional (responsabilidad profesional y compromiso ético } \\
\text { con el ejercicio de la profesión a favor de lo colectivo y de la asistencia universal) }\end{array}$ & 98,2 \\
\hline $\begin{array}{l}\text { Investigación (respeto que se debe de tener con los pacientes, sujeto de investigación) } \\
\text { a La suma del porcentaje puede ser mayor del 100\% porque la misma respuesta podría clasificarse en más de una categoría. La frecuencia } \\
\text { relativa se calculó sobre el total de } 34 \text { participantes. }\end{array}$ & 96,5 \\
\hline
\end{tabular}

dades de preferencias cognitivas y psicomotoras-, representa una actitud en la formación en Odontología que contribuye a una percepción más consciente y crítica de la realidad [20]. Eso puede ser visto como una actitud que va a contramano del modelo dominante de enseñanza o como una visión de innovación alienante, que procura explorar nuevas alternativas que se abren al conflicto y a las contradicciones, pudiendo ser un marco relevante para la construcción de nuevas posibilidades en la enseñanza [21]. Sin embargo, esa reflexión fomentada por la bioética puede generar posibles impactos en el día a día profesional de los titulados.

Saliba et al [22], en su estudio sobre los métodos de evaluación empleados en la graduación de la Facultad de Odontología de Araçatuba-UNESP, observaron que el 99,3\% de las asignaturas usan principalmente la prueba escrita. Rozendo et al [23] mencionaron que la prueba escrita fue utilizada por el $79,3 \%$ de los profesores participantes. No obstante, en este estudio, todos los profesores emplean la prueba escrita en la asignatura de bioética (100\%).

La utilización de la prueba teórica puede ser explicada por el hecho de que, en todo el sistema educacional superior brasileño, la evaluación del estudiante se orienta hacia la retención de conocimientos factuales [24-26]. Con este método no se puede desarrollar una conciencia crítica del futuro profesional -lo que la asignatura de bioética busca fomentar-, permaneciendo una inmadurez de toma de decisión posterior [27].
Entre otros instrumentos de evaluación, Rozendo et al [23] citan la observación del desempeño del alumno en clase $(51,7 \%)$, el trabajo escrito $(31,0 \%)$, el seminario $(31,0 \%)$ y la prueba práctica $(20,7 \%)$. No obstante, en esta investigación, el trabajo escrito y los seminarios (75\%), la prueba práctica y debates (25\%), y la asiduidad/puntualidad $(13,9 \%)$ también se utilizaron como forma de evaluación. Sin embargo, Saliba et al [22], en estudio con académicos, concluyeron que en la percepción de éstos, los seminarios $(27,9 \%)$ presentaban una menor contribución a la formación académica y profesional. El principal motivo de la baja adopción de seminarios como método para facilitar el aprendizaje tal vez se deba a la participación desigual entre los componentes, lo que acarrea desinterés y descontento con los resultados obtenidos, y ocasiona falta de conocimiento de la materia en algunos alumnos. Ante eso, todos los métodos de evaluación deben analizarse para una posible reformulación o incluso deben excluirse si no se alcanzan sus objetivos.

Por tanto, para ejercer la práctica de salud, el profesional debe concienciarse de que hay varios factores relacionados con ella -orgánicos, psicológicos, socioeconómicos y espirituales- que deberán comprenderse y analizarse conjuntamente [28]; es lo que la asignatura de bioética debe fomentar en los alumnos, ya que se caracteriza por ser una asignatura reflexiva, polémica y actual.

Siqueira [29] menciona que el instrumento pedagógico más adecuado para la enseñanza de 
la bioética es el de las actividades de enseñanza con pequeños grupos de estudiantes (en promedio, ocho alumnos), que recurren a procesos interactivos y participativos y consideran todas las variables del problema presentado, sobre todo el debate; ello concuerda con el $25 \%$ de los docentes entrevistados en la presente investigación, que emplean este medio como método de evaluación de la asignatura de bioética.

En la literatura odontológica brasileña se observan pocas referencias bibliográficas sobre bioética y odontología, específicamente de libros. Se observa también que aún es tímida esa expresión de la bioética en la odontología, pues todas las referencias mencionadas de libros son apenas capítulos. Actualmente existen numerosas referencias de artículos y trabajos científicos odontológicos dirigidos al área de bioética, como han citado diversos entrevistados. Por tanto, se hacen necesarias bibliografías relacionadas específicamente con la odontología, una vez que la mayoría de los docentes emplea referencias médicas, lo que muchas veces no corresponde a la realidad del cirujano dentista.

Sobre el hecho de que la asignatura de bioética se relacione con otras asignaturas, un $74,2 \%$ citó que ésta se relaciona directa o indirectamente con todas las otras asignaturas, pues el factor innovador de la bioética es ser un área del conocimiento que engloba varios temas, ya que presenta una concepción de interdisciplinaridad o incluso transdisciplinaridad [30]. Además, aunque la clínica pueda suplir las necesidades individuales y privadas con calidad técnica reconocida, hay un desafío y una distancia entre la enseñanza teórica de la odontología y la perspectiva de universalización de la práctica en salud bucal en relación con las demandas de la realidad brasileña [31], ámbito en el cual la bioética ayuda con su reflexión al futuro profesional.

Respecto a la importancia de la asignatura de bioética en la formación del cirujano dentista, un $64,7 \%$ enfatizó la importancia de su enseñanza en la relación profesional-paciente. En todos los escenarios de práctica profesional, los problemas son una realidad y la bioética favorece la reflexión y propone los cambios necesarios para superar la indignación cotidiana [3]. Por eso, la interdisciplinaridad se contempla como una posibilidad de solventar distorsiones provocadas por el exceso de especialización, que genera la fragmentación del conocimiento.
Según Galindo [32], la enseñanza y reflexión de la bioética en los cursos de Odontología posibilita la preponderancia del paradigma humanitario sobre el paradigma financiero, científico y jurídico, además de favorecer el equilibrio entre los intereses de la sociedad y los del individuo, aunque se encuentre en la posición de profesional de la salud o de paciente. Además, el objetivo de la enseñaza y de la discusión bioética en la odontología consiste en contribuir a la formación integral del futuro profesional, orientándolo hacia una práctica profesional pautada por la ética, principios, reglas, virtudes generales y comunes a las diferentes áreas de las ciencias de la salud, enfatizando la visión del paciente como un todo y contribuyendo a una correcta relación profesional-paciente [33].

La bioética presenta una metodología de enseñanza-aprendizaje y prácticas de evaluación conservadoras; se necesitan otras metodologías dirigidas a la reflexión de los problemas actuales en el área de odontología, que contribuyan significativamente a la formación integral del cirujano dentista.

\section{Bibliografía}

1. Brasil. Ministério da Educação e Cultura. Conselho Nacional de Educação. Resolução CNE/CES 3/2002. Diário da União, 4 de marzo de 2002. URL: http://dtr2002. saude.gov.br/sesu/ftp/resolucao/03020odontologia.doc. [08.06.2006].

2. Segre M. O ensino da bioética. Mundo Saúde 2005; 29: 436-43.

3. Araújo ME. Palavras e silêncio na educação superior em Odontologia. Ciência \& Saúde Coletiva 2006; 11: 179-82.

4. Noro LRA, Albuquerque DF, Ferreira MEM. O desenvolvimento do processo ensino aprendizagem: visão do aluno e do professor. Rev ABENO 2005; 6: 109-14.

5. Heinzen JLN. A avaliação institucional na UDESC: visualizando o fluxograma processual. Aval 1997; 2: 19-24.

6. Soares EC, Gilberto IC. Avaliação - para que? Rev Bras Educ Med 1990; 14: 11-6.

7. Troncon LEA. Responses of students and faculty members to changes in assessment in a Brazilian medical school. In Rothman AI, Cohen R, eds. Proceedings of the Sixth Ottawa conference on Medical Education. Toronto: Uni- 
versity of Toronto Bookstore Custom Publishing; 1995. p 120-2.

8. De Ketele JM, Roegiers X. Metodologia da recolha de dados. Lisboa: Instituto Piaget; 1999.

9. Bardin L. Análise de conteúdo. Lisboa: Edições 70; 1994.

10. Gonçalves ER, Verdi MIM. os problemas éticos no atendimento a pacientes na clínica odontológica de ensino. Ciência \& Saúde Coletiva 2007; 12: 755-64.

11. Lakatos E, Marconi M. Fundamentos de metodologia científica. São Paulo: Editora Atlas; 1985.

12. Fox D. El proceso de investigacion en educacion. 2 ed. Pamplona: Ediciones Universidad de Navarra; 1987.

13. Pinheiro A, Silva B. A estruturação do processo de recolha de dados on-line. In: Actas da X Conferência Internacional Avaliação Psicológica, Formas e Contextos. Braga: Psiquilíbrios Edições; 2004. p. 522-9.

14. Zilbovicius C. A saúde bucal e o sistema único de saúde: integralidade seletiva em uma conjuntura neoliberal [tesis doctoral]. São Paulo: Universidade de São Paulo; 2005

15. Santos JC, Rumel D. Emergência médica na prática odontológica no estado de Santa Catarina: ocorrência, equipamentos e drogas, conhecimento e treinamento dos cirurgiões-dentistas. Ciência \& Saúde Coletiva 2006; 11: 183-90.

16. Musse JO, Boing AF, Martino FS, Silva RHA, Vaccarezza $\mathrm{GF}$, et al. $\mathrm{O}$ ensino da bioética nos cursos de graduação de odontologia do estado de São Paulo. Arq Cienc Saúde 2007; 14: 13-6.

17. Segre M. O ensino da bioética lato sensu. Bioética 2003; 11: $57-60$

18. Carvalho AS, Oswaldo W. Publicações portuguesas em bioética. Bioética 2003; 11: 61-72.

19. Silva JR. O ensino da bioética nas faculdades de medicina portuguesas. Bioética 2003; 11: 43-50.

20. Prado MM, Garrafa V. A bioética na formação em odontologia: importância para uma prática consciente e crítica. Comum Cienc Saúde 2006; 17: 263-74.

21. Cyrino EG, Rizzato ABP. Contribuição à mudança curricular na graduação da Faculdade de Medicina de Botucatu. Rev Bras Saúde Matern Infant 2004; 4: 59-69.
22. Saliba NA, Moimaz SAS, Rapfael HS, Foster NF, Gonçalves PE. Métodos de avaliação de aprendizagem empregados no curso de graduação de odontologia. Rev Odontol UNESP 2008; 37: 79-83.

23. Rozendo CA, Casagrande LDR, Schneider JF, Pardin LC Uma análise das práticas docentes de professores universitários da área de saúde. Rev Latinoam Enferm 1999; 7: 15-23.

24. Troncon LEA, Picinato CE, Cianflone ARL. Implantação de um programa de avaliação terminal do desempenho dos graduandos para estimar a eficácia do currículo da Faculdade de Medicina de Ribeirão Preto. Rev Assoc Med Brás 1999; 45: 217-24.

25. Troncon LEA. Avaliação terminal de competências clínicas dos graduandos em medicina: relato da experiência inicial da Faculdade de Medicina de Ribeirão Preto. Ribeirão Preto: Faculdade de Medicina de Ribeirão Preto; 1996.

26. Vianna HM. Avaliação educacional nos 'cadernos de pesquisa'. Cad Pesq São Paulo 1992; 80: 100-5.

27. Simões RHS. Da avaliação da educação à educação da avaliação: o lugar do(a) educador(a) no processo da avaliação da pós-graduação no Brasil. Psicol Soc 2004; 16: 124-34.

28. Pereira ALF. As tendências pedagógicas e a prática educativa nas ciências da saúde. Cad Saúde Publica 2003; 19: 1527-34.

29. Siqueira JE. O ensino da bioética no curso médico. Bioética $2003 ; 11: 33-42$.

30. Azevedo EES. Ensino de bioética: um desafio transdiciplinar. Interface Comum Saúde Educ 1998; 2: 127-37.

31. Secco LG, Pereira MLT. Formadores em odontologia: profissionalização e desafios político-estruturais. Ciência \& Saúde Coletiva 2004; 9: 113-20.

32. Galindo GC. Uma manera muy complicada de hablar de bioética a los odontólogos. Universitas Odontológicas 2001; 21: 3-5

33. Marcos F. Enseñanza de bioética en odontología. Rev Assoc Odontol Argent 2004; 92 : 123-7 